\title{
Potential hydrodynamic origin of frictional transients in sliding mesothelial tissues
}

\author{
Stephen H. LORING ${ }^{1, *}$, James P. BUTLER ${ }^{2}$ \\ ${ }^{1}$ Department of Anesthesia, Critical Care and Pain Medicine, Beth Israel Deaconess Medical Center and Harvard Medical School, Boston \\ MA 02215, USA \\ ${ }^{2}$ Department of Medicine, Division of Sleep Medicine, Brigham and Women's Hospital and Harvard Medical School, Boston MA 02215, USA \\ Received: 26 January 2013 / Revised: 26 April 2013 / Accepted: 20 May 2013 \\ C The author(s) 2013. This article is published with open access at Springerlink.com
}

\begin{abstract}
Steady-state and transient variations in frictional force observed in tribological experiments of mesothelial tissues sliding in lubricant were analyzed with a mathematical model to test the hypothesis that such phenomena are manifestations of elastohydrodynamic lubrication and, importantly, do not require physical contact between the sliding surfaces. The model incorporates three phenomena characteristic of elastohydrodynamic lubrication: thinning of the liquid layer between sliding surfaces under a normal load ("squeeze-out"), thickening of the liquid layer due to hydrodynamic pumping, and smoothing of the elastic surfaces caused by hydrodynamic pressure gradients. Observations in soft mesothelial tissues sliding in lubricant showed variations in steady state friction with velocity, load, and lubricant viscosity. In non-steady sliding, the decay rate of frictional transients at the start of rotation varied with velocity, the amplitudes of these transients varied with the preceding periods without rotation, and frictional force varied during sinusoidal sliding. Model simulations were qualitatively similar to experimental results, supporting these mechanisms. Higher lubricant viscosity increased lubricating layer thickness and lowered friction at low speeds and increased friction at high speeds, supporting hydrodynamic pumping. We conclude that the frictional variations seen with sliding mesothelial tissues are consistent with elastohydrodynamic lubrication without contact between the sliding surfaces.
\end{abstract}

Keywords: pleural space; breathing; pericardium; lubrication; model

\section{Introduction}

Throughout life, the mesothelial surfaces within the body slide against each other, lubricated by a thin layer of serous fluid. The relationships between frictional force and tribological parameters such as sliding velocity, normal load, and viscosity have been used to infer the nature of the physical interactions between these tissues [1-5]. For example, D'Angelo et al. [4] studied pleural tissues during oscillatory rectilinear sliding and found the friction coefficient, defined as the ratio of shear stress to normal stress on the sliding surfaces, to be nearly independent of the frequency

* Corresponding author: Stephen H. LORING.

E-mail: sloring@bidmc.harvard.edu of oscillation at a constant displacement amplitude. They concluded that this independence with respect to velocity was consistent with boundary lubrication in which contact or near-contact between asperities bears the normal load [4]. By contrast, in a subsequent study using a rotating tribometer that measured the friction coefficient as torque divided by normal load, Loring et al. [5] found that the friction of wetted pleural tissues on a rotating plate varied with rotation rate and hence circumferential velocity, which is inconsistent with boundary lubrication but consistent with mixed elastohydrodynamic or fully-developed hydrodynamic lubrication, in which hydrodynamic pressure in the fluid bears much or all of the normal load. In a similar rotational apparatus, Lin et al. [6] measured the thickness of the fluid layer 
between sliding mesothelial tissues and a rotating glass plate and found that the thickness of the fluid layer beneath the tissue surface depended on sliding velocity. In particular, higher velocities resulted in greater fluid thickness and a faster approach to the steady state fluid thickness, consistent with hydrodynamic lubrication. In addition, soft materials sliding in lubricant are smoothed by hydrodynamic pressure gradients, making the lubricant layer more uniform in thickness [7]. Considered together, the latter three studies suggest that soft materials with statically uneven surfaces sliding in lubricants are smoothed and deformed so as to become load-bearing, thus maintaining a layer of lubricant between sliding surfaces $[8,9]$.

The latter studies and subsequent observations suggest that the tribological behavior of sliding tissue samples in these experiments can be largely explained by the following three phenomena characteristic of elastohydrodynamic lubrication. (1) Squeeze-out: thinning of the liquid layer between the sliding surfaces due to centrifugal flow driven by load-dependent pressure gradient. An example of this effect in vivo is the flow of pleural liquid away from the region between a convex rib indenting the lung towards the surrounding regions of the pleural space where the normal stress and fluid pressure are lower. (2) Hydrodynamic pumping: thickening of the fluid layer between the sliding surfaces due to centripetal fluid flow driven by local hydrodynamic pressure gradients caused by the sliding of slightly uneven surfaces that are nearly, but not completely, parallel. This phenomenon is responsible for load support in hydrodynamic lubrication. An example of this effect in vivo is the hydrodynamically driven flow of pleural fluid from a relatively thick layer of liquid near a lobar margin into the pleural space covering the costal surface of the lung [10]. (3) Elastohydrodynamic smoothing: reversible deformation and flattening of the sliding surfaces caused by hydrodynamic pressure gradients that result in a decrease in the spatial nonuniformity of thickness of the fluid layer. An example of this effect in vivo is the small-scale reversible deformation that would smooth mesothelial surfaces wherever they slide relative to each other in the presence of serous fluid.
In this report, examples of steady state and transient friction observed experimentally in vitro are presented and then simulated with a mathematical model that synthesizes the three phenomena above: thinning of the fluid layer due to squeeze-out, thickening due to hydrodynamic pumping, and elastohydrodynamic smoothing. The model is based on geometrical and physical parameters of our experimental preparations, variations in velocity similar to those in the experiments, and a free parameter relating the velocity of sliding to the magnitude of the hydrodynamic pressure opposing squeeze-out. Simulations were compared with experimental results to quantify the importance of these mechanisms to the transient phenomena observed under different experimental conditions.

\section{Methods}

\subsection{Experimental preparation and apparatus}

Tribological experiments on mesothelial tissues were conducted using the peritoneal surface of rat belly wall sliding on a rotating glass plate (Fig. 1) as described previously $[5,6]$ or on a rotating plate covered with tissue. Briefly, a tissue sheet consisting of the inner layer of the abdominal wall, including the peritoneum and attached muscle layers, was dissected free and mounted, mesothelial side outward, at the mouth of a shallow tissue-cup ( $3.2 \mathrm{~cm}$ diameter). In the top-fixed experimental apparatus [5], the mesothelial tissue and cup, mounted on a shaft and bearing for measurement of rotational torque, were held close $(<1 \mathrm{~mm})$ to the surface of a rotating glass plate lubricated with physiologic saline. The tissue sheet was then pressed against the glass plate using air pressure within the tissue cup, thus applying a known normal stress on the sliding mesothelial surface. Data from those experiments were reanalyzed to characterize the transient changes in torque at the onset of motion. In new experiments using a top-weighted apparatus, the torque measuring apparatus was mounted on a balance arm and pressed against the rotating glass plate by an applied weight. With this apparatus, friction between tissue surfaces was also measured by mounting mesothelial tissue, prepared as above, on a rotating platform slightly larger than the stationary tissue and cup. 


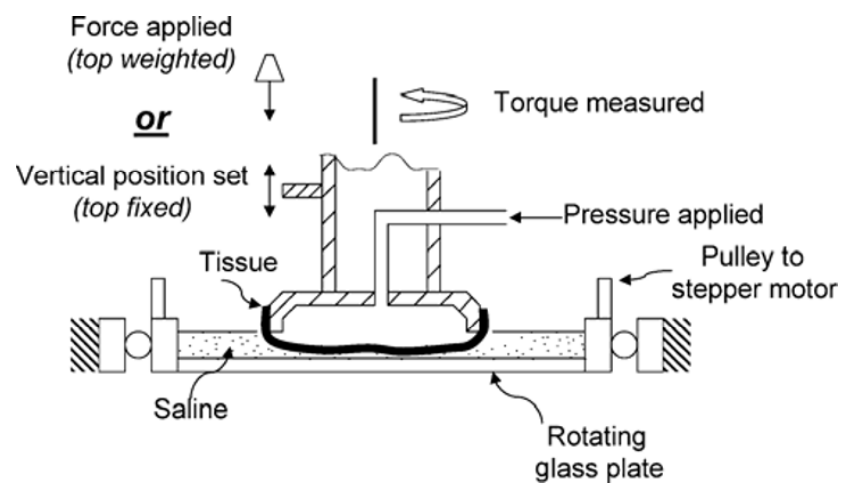

Fig. 1 Schematic of apparatus illustrating some of the features of the rotational tribometers used in the experiments.

The glass plate or tissue platform was rotated by a computer-controlled stepper motor, and the applied pressure, torque, and angular displacement of the drive system were recorded at $>750 \mathrm{~Hz}$ (Dataq Instruments, Akron, $\mathrm{OH})$. Friction was quantified by rotational torque measured with a strain gauge [5] and divided by normal load to produce a rotational coefficient of friction (CF). Details of individual experiments are described in Section 3.

\subsection{Model simulation}

A mathematical model was used to simulate the experiments and explore mechanism. The model incorporates a disc of soft tissue with an undulating bottom surface pressed against a flat plate rotating in the presence of lubricating fluid (Fig. 2). The unevenness of the bottom tissue surface decreases as the tissue approaches the opposing plate. The parameters of the model are similar to those of the experiment and are presented in Table 1. Experimental input variables include rotation rate and normal pressure load as functions of time. Output variables include frictional force (quantified by torque) and fluid thickness. The dynamic behavior of the system is determined by the three phenomena introduced above and further defined below: (1) thinning of the fluid layer due to squeezeout of fluid caused by the normal load, (2) thickening of the fluid layer due to hydrodynamic pumping of fluid from the surrounding reservoir, and (3) elastic deformation and flattening of the surface unevenness causing elastohydrodynamic smoothing of the tissue surface, decreasing the amplitude of surface roughness.

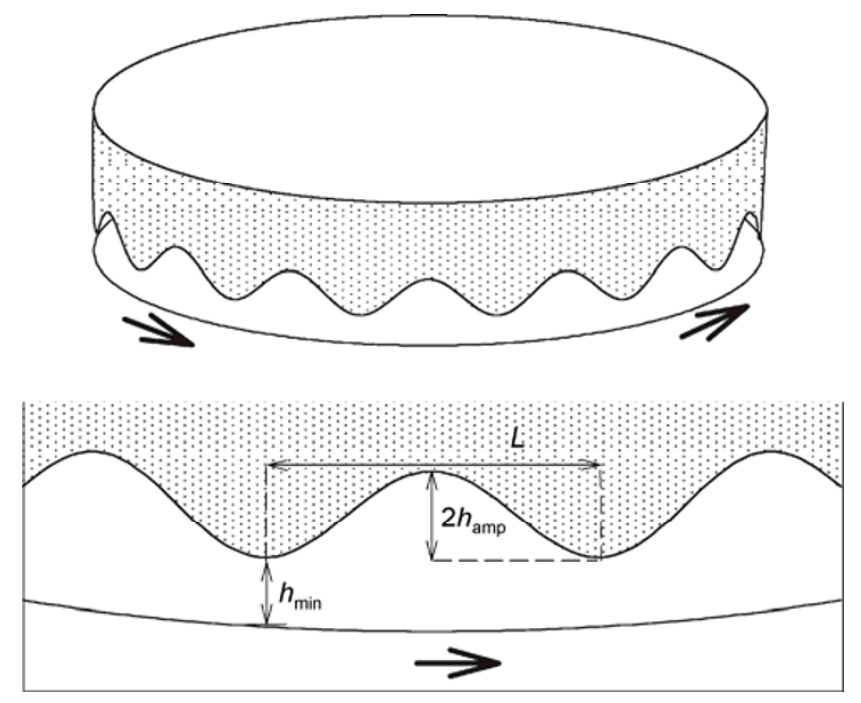

Fig. 2 Model of a soft tissue disk with an uneven bottom surface pressed by a normal load against a rotating flat disk, all bathed in lubricant. The minimum fluid thickness $\left(h_{\min }\right)$, amplitude of unevenness $\left(h_{\mathrm{amp}}\right)$ and characteristic wavelength $(L)$ are not to scale.

Table 1 Constants of the model simulations.

\begin{tabular}{clc}
\hline Constant & \multicolumn{1}{c}{ Description } & Value \\
\hline$R_{\text {disk }}$ & disk radius ( $R$ in the equations above) & $1.5 \mathrm{~cm}$ \\
$h_{\text {amp }}^{0}$ & $\begin{array}{l}\text { initial undeformed amplitude of uneven- } \\
\text { ness }\end{array}$ & $70 \mu \mathrm{m}$ \\
$L$ & wavelength of unevenness & $1000 \mu \mathrm{m}$ \\
$\mu$ & lubricant viscosity & 0.01 \\
& & poise \\
$\mathrm{k}_{\text {pump }}$ & constant of hydrodynamic pumping & 100 \\
$h_{\text {min, crit }}$ & $h_{\text {min }}$ below which $P_{\text {hydro }}$ progressively & $8 \mu \mathrm{m}$ \\
& decreases
\end{tabular}

${ }^{*}$ Parameters used for fitting simulation to experimental data

\subsection{Mechanisms of hydrodynamic lubrication}

\subsubsection{Squeeze-out}

Squeeze-out is based on the physics of a piston sinking under a normal load in lubricant toward a flat surface (see Eq. (9) below). The normal load is balanced by the effective hydrodynamic pressure caused by downward movement of the piston. The rate of descent (fluid thinning) is directly proportional to the load and inversely proportional to fluid viscosity, the cube of the harmonic mean fluid layer thickness, and the radius of the disk to the 4 th power. 


\subsubsection{Hydrodynamic pumping}

The load-supporting pressure due to hydrodynamic pumping is added to the hydrodynamic pressure due to squeeze-out. When the pressure due to the normal load is greater than that produced by hydrodynamic pumping, fluid flow is centrifugal (from disk center) and the fluid layer gets thinner. Conversely, when the hydrodynamic pumping pressure is greater than the normal load, fluid flow is centripetal and the fluid layer gets thicker. Hydrodynamic pumping pressure is the global effect of highly local hydrodynamic pressures generated between sliding surfaces that are nearly but not completely parallel. Consistent with the major features of lubrication theory [11], hydrodynamic pumping is assumed to be proportional to sliding velocity and fluid viscosity and inversely proportional to the minimum fluid thickness, $h_{\min }$. To simulate the increase in CF at very high sliding speeds, hydrodynamic pumping pressure is assumed to diminish as the fluid thickness gets large compared with the characteristic wavelength of the surface unevenness.

\subsubsection{Elastohydrodynamic smoothing}

Relative flattening of the surface undulations due to hydrodynamic pressure is assumed to occur as the uneven tissue approaches the flat plate. The amplitude $\left(h_{\mathrm{amp}}\right)$ of the unevenness of the tissue disk remains relatively constant near its initial undeformed amplitude $h_{\text {amp }}^{0}$ when the mean fluid thickness $(h)$ is large and decreases smoothly and progressively as the minimum thickness $h_{\min } \rightarrow 0$, or equivalently, as $h$ approaches $h_{\mathrm{amp}}^{0}$. The harmonic mean thickness $\left(h_{\mathrm{hm}}\right)$ and $h_{\min }$ of the fluid are derived from $h$ and $h_{\text {amp }}^{0}$. Flattening of the surface to an extreme degree is assumed to reduce hydrodynamic pumping, leading to an increase in $\mathrm{CF}$ at very low sliding speeds.

Simulations of the model, including fluid thickness and coefficient of rotational friction, were compared to experimental data obtained with the following velocity-time protocols: (1) steady-state sliding at different constant rates, loads, and lubricant viscosities; (2) a step increase in rotation rate from zero to various constant speeds following $30 \mathrm{~s}$ without motion; (3) constant speed rotation at different rates interrupted by different periods without rotation; and (4) sinusoidal rotation at various frequencies and peak rotation rates.

\subsection{Model details}

Conceptually, the model is a disk of tissue with an uneven bottom surface pressed against a coaxial rotating flat surface, the "bottom plate" (Fig. 2). The flow of fluid within the space between tissue disk and bottom plate depends on the pressure gradient caused by the combined effects of the normal force (load) applied to the tissue disk, which causes centrifugal flow and fluid layer thinning (squeeze-out) and the pressure generated by hydrodynamic pumping, which causes centripetal flow and fluid layer thickening. When the tissue is pressed close to the bottom plate, it is smoothed (flattened) by normal stresses caused by hydrodynamic pressures. Whereas the physics underlying squeeze-out is well understood and a rigorous solution exists, the physics of hydrodynamic pumping and soft tissue smoothing are only partially understood, and a rigorous solution in general is lacking.

The theoretical basis for the model starts with a dynamical argument to find the relationship between normal force, $F_{n}$, in relation to the rate of change in the height of the fluid channel between the disk and the plate. In what follows, we assume circular symmetry and deal only with the radial dependence of variables.

The volume flow rate (or flux) of fluid inward at any radius $r$ is

$$
\dot{Q}(r)=\pi r^{2} \frac{\mathrm{d} h}{\mathrm{~d} t}
$$

where $h$ is the mean height of the tissue surface above the plate. In particular, the flow rate, $\dot{Q}$, into the region between the disk and the bottom plate from the reservoir is

$$
\dot{Q}=\dot{Q}(R)=\pi R^{2} \frac{\mathrm{d} h}{\mathrm{~d} t}
$$

where $R$ is the radius of the tissue disk.

The cylindrical cross-sectional area of the fluid channel at radius $r$ is $A(r)=2 \pi r h$, and so the mean fluid radial velocity $\bar{U}(r)$ is given by

$$
\bar{U}(r)=\frac{\dot{Q}(r)}{A(r)}=\frac{r}{2 h} \frac{\mathrm{d} h}{\mathrm{~d} t}
$$


This is used at each $r$ to determine $\frac{\mathrm{d} P}{\mathrm{~d} r}$, the pressure gradient driving flow in the channel. In steady state, the fully developed fluid velocity is quadratic in height, $y$, and vanishes at the bottom and top surfaces secondary to a no slip condition. Thus, $U(y, r)=U(r)_{\max } \frac{4}{h^{2}} y(h-y)$, where $U(r)_{\max }$ is the velocity at mid-channel. The average velocity $\bar{U}(r)=$ $\frac{1}{h} \int_{0}^{h} U(y, r)=\frac{2}{3} U_{\max }(r)$, and so, from Eq. (3), we have

$$
U_{\max }(r)=\frac{3 r}{4 h} \frac{\mathrm{d} h}{\mathrm{~d} t}
$$

The gradients in $U(y, r)$ with respect to $y$ induce a viscous drag on the boundary, which must be balanced by radial gradients in pressure. The force on a differential volume of fluid between $r$ and $r+\mathrm{d} r$ of angular width $\mathrm{d} \vartheta$ due to the two boundaries is thus twice product of the shear stress and the area, given by $-2 \mu \mathrm{d} U /\left.\mathrm{d} y\right|_{y=0} r \mathrm{~d} r \mathrm{~d} \vartheta=-\left(8 \mu U_{\max } / h\right) r \mathrm{~d} r \mathrm{~d} \vartheta$. The force on the differential element due to a pressure gradient is simply $h r \mathrm{~d} \vartheta \mathrm{d} P$. Equating these yields

$$
\mathrm{d} P / \mathrm{d} r=-6 \mu \frac{r}{h^{3}} \frac{\mathrm{d} h}{\mathrm{~d} t}
$$

The pressure at any radius is

$$
P(r)=P_{0}+\int_{R}^{r} \frac{\mathrm{d} P}{\mathrm{~d} r}(r)=P_{0}-\frac{3 \mu}{h^{3}} \frac{\mathrm{d} h}{\mathrm{~d} t}\left[R^{2}-r^{2}\right]
$$

where $P_{0}$ is pressure in the surrounding reservoir. The normal force (load) is

$$
F_{\mathrm{n}}=2 \pi \int_{0}^{R} P(r) r \mathrm{~d} r=-\frac{3 \pi \mu R^{4}}{2 h^{3}} \frac{\mathrm{d} h}{\mathrm{~d} t}
$$

and therefore

$$
\frac{\mathrm{d} h}{\mathrm{~d} t}=-\frac{2 h^{3} F_{\mathrm{n}}}{3 \pi \mu R^{4}}
$$

or, in terms of average pressure $(\bar{P})$,

$$
\frac{\mathrm{d} h}{\mathrm{~d} t}=-\frac{2 h^{3} \bar{P}}{3 \mu R^{2}}
$$

The model simulations are based on Eq. (9). The rate of change in thickness of the fluid layer is a function of the effective average pressure, i.e., the sum of the average pressure load, acting to decrease thickness, and a hydrodynamic pressure $\left(P_{\text {hydro }}\right)$ due to sliding of the uneven tissue surface against the rotating disk, acting to increase thickness. $P_{\text {hydro }}$ is assumed to have the same spatial distribution as that of the pressure load and to oppose the gradients caused by the load or downward movement of the tissue disk. Constants of the model include the disk radius $(R)$ and fluid viscosity $(\mu)$.

The pressure load and rotation rate $(f$, signed such that $f>0$ when rotation is counterclockwise, typical units are revolutions per second) are input variables. Hydrodynamic pressure caused by tissue sliding is due to wedge-like deformations of these otherwise symmetrical undulations of the tissue surface of amplitude, $h_{\text {amp }}$. The wavelength $(L)$ and initial undeformed amplitude of microscopic sinusoidal unevenness of the tissue surface $\left(h_{\text {amp }}^{0}\right)$ are specified. When $h>>h_{\text {amp }}^{0}, h_{\text {amp }} \cong h_{\text {amp }}^{0}$. As the tissue approaches the bottom plate, $h_{\text {amp }}$ is reduced by adjusting the local minimum thickness of the liquid layer $\left(h_{\min }=h-h_{\text {amp }}\right)$ to maintain $h_{\min }>0$ according to the following equation,

$$
h_{\min }=\frac{h}{1+h_{\mathrm{amp}}^{0} / h} .
$$

The rate of increase or decrease in the average $h$ (Eq. (9)) is calculated using the harmonic mean thickness $h_{\mathrm{hm}}$, given by

$$
1 / h_{\mathrm{hm}}=1 / 2\left(1 / h_{\min }+1 / h_{\max }\right),
$$

where $1 / 2\left(h_{\max }+h_{\min }\right)=h$. The equation for $P_{\text {hydro }}$ is a simplification based on lubrication theory [11]. In essence, the normal (lifting) force is assumed to be proportional to velocity, viscosity, the area of the uneven sliding surface, and roughly inversely proportional to the minimum fluid thickness,

$$
P_{\text {hydro }}=\frac{\mathrm{k}_{\text {pump }} \mu|f| 2 \pi R^{2}}{h_{\min }},
$$

where $k_{\text {pump }}$ is a constant. We modified the formula to simulate a reduction of lift when the tissue surface is far separated from the disk $\left(h>>h_{\text {amp }}\right)$, 


$$
P_{\text {hydro }}=\frac{\mathrm{k}_{\text {pump }} \mu|f| 2 \pi R^{2}}{h_{\text {min }}} \cdot \frac{L}{L+h_{\text {min }}},
$$

where $L$ is the undulation wavelength. We further modified this formula to simulate a reduction in lift when the undulations in the tissue surface flatten in close proximity to the bottom plate,

$$
P_{\text {hydro }}=\frac{\mathrm{k}_{\text {pump }} \mu|f| 2 \pi R^{2}}{h_{\text {min }}+\mathrm{h}_{\text {min, crit }} h_{\min }^{0.25}} \cdot \frac{L}{L+h_{\text {min }}},
$$

where $h_{\text {min, rit }}$ is a constant. Eq. (9) is integrated to determine changes in $h$.

Shear stress on a differential area $(\mathrm{d} S)$ of the disk (based on $h_{\mathrm{hm}}$ ) is

$$
\mathrm{d} S=\frac{\mu}{h_{\mathrm{hm}}} r f,
$$

which is integrated to obtain torque

$$
\text { Torque }=\frac{\mu}{h_{\mathrm{hm}}} \int_{0}^{R} r f S_{r} \mathrm{~d} r=\frac{\pi^{2} \mu f R^{4}}{h_{\mathrm{hm}}} .
$$

\section{Results}

\subsection{Steady state rotation at constant rates: effects of velocity, load, and viscosity}

\subsubsection{Experimental: tissue sliding on tissue, effects of} rotation rate and load

Seven experiments were performed with the topweighted apparatus with tissue rotating on tissue under loads of 5 or $10 \mathrm{~g}$, the upper tissue disk being pressurized with 100 or $200 \mathrm{~Pa}$. The bottom tissue surface was rotated at various rates in alternating directions with a square-wave pattern that allowed steady state CF determinations after transients died out. The coefficient of friction (torque/load) vs. velocity curves were reminiscent of classical Stribeck curves, with relatively high $\mathrm{CF}$ at the lowest rotation rates, lower $\mathrm{CF}$ at transitional rates and increasing $\mathrm{CF}$ at higher rates, but there was substantial variability among experiments, both in the magnitude of $\mathrm{CF}$ and the pattern of its variation with velocity. These results were similar to those of tissue sliding on glass published previously using the top-fixed apparatus [5]. CF did not vary systematically with load in these experiments (Fig. 3). In general, the tribological behavior of tissue sliding on tissue was similar to that of tissue sliding on glass, suggesting a common mechanism.

\subsubsection{Experimental: tissue sliding on glass, effects of} rotation rate and viscosity

Seven experiments were performed with the topweighted apparatus with tissue rotating on flat glass under loads of 0.10 or $0.15 \mathrm{~N}$, with the tissue disk pressurized with 200 or $300 \mathrm{~Pa}$. The CF measured with saline lubricant, viscosity $\sim 1$ centipoise (cp), was compared to that with solutions of 4.0 or $6.5 \mathrm{mg} / \mathrm{ml}$ carboxymethylcellulose in saline, viscosity $\sim 0.020$ or $\sim 0.033 \mathrm{cp}$ (measured with a U-tube viscometer). At the lowest rotation rates, CF tended to be lower with the higher viscosity lubricant, whereas at the highest rotation rates, the opposite was true (Fig. 4).

\subsubsection{Model simulations and mechanism: effects of velocity, load, and viscosity}

In steady state simulations (Fig. 5), CF is relatively constant at lower velocities, decreasing slightly at intermediate speeds and increasing at higher speeds, reproducing the experimental changes in CF with speed.

The mechanism of $\mathrm{CF}$ variation in the simulation can be related to the changes in $h$ at low and high velocities. In lubrication theory and in our model, the normal force supporting the load above a wedge sliding with velocity $U$ and the shear force both vary roughly as $h^{-1}$. In our simulations, $h$ varies as $U^{0.4}$ throughout the range of velocities studied. At very low velocities, decreases in velocity cause fractional decreases in $h$ that re-establish the steady state hydrodynamic pressure to balance the normal load without causing large changes in shear stress. When $h$ approaches $h_{\text {amp }}^{0}$, hydrodynamic smoothing degrades the hydrodynamic pumping, causing a large decrease in $h$ and consequent increase in CF at very low speeds. Conversely, at very high speeds, increases in velocity cause relatively small fractional increases in $h$, and shear force increases with speed.

Figure 5 also shows the effects of lubricant viscosity and load. Doubling of the load decreases $h$ and increases CF at low speeds while slightly reducing CF 

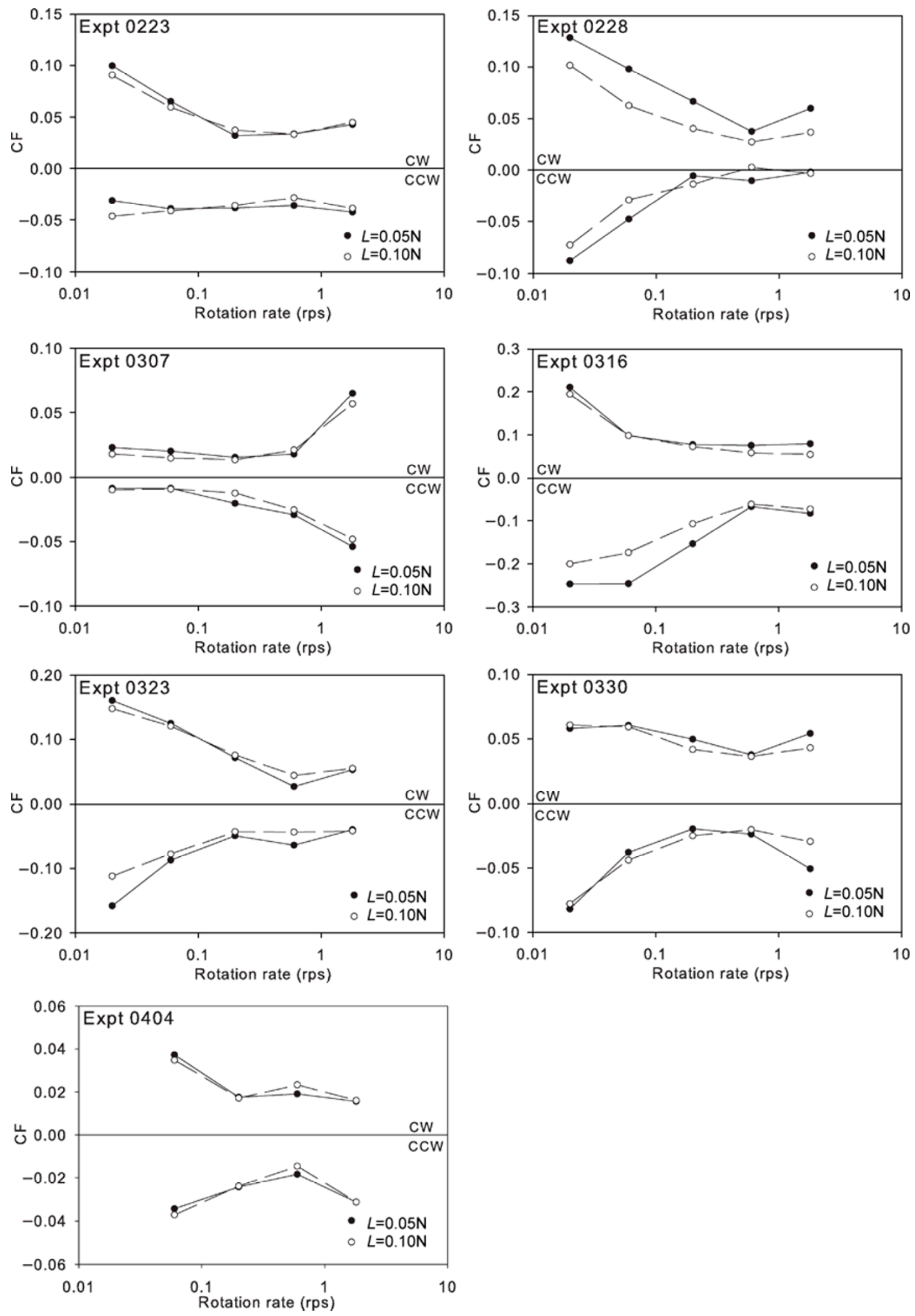

Fig. 3 Coefficient of friction (CF) of tissue rotating on tissue. Rotation was alternating clockwise (CW) and counter-clockwise (CCW, CF shown as negative) under loads $(L)$ of 0.05 or $0.10 \mathrm{~N}$ (See Section 3.1.1) 

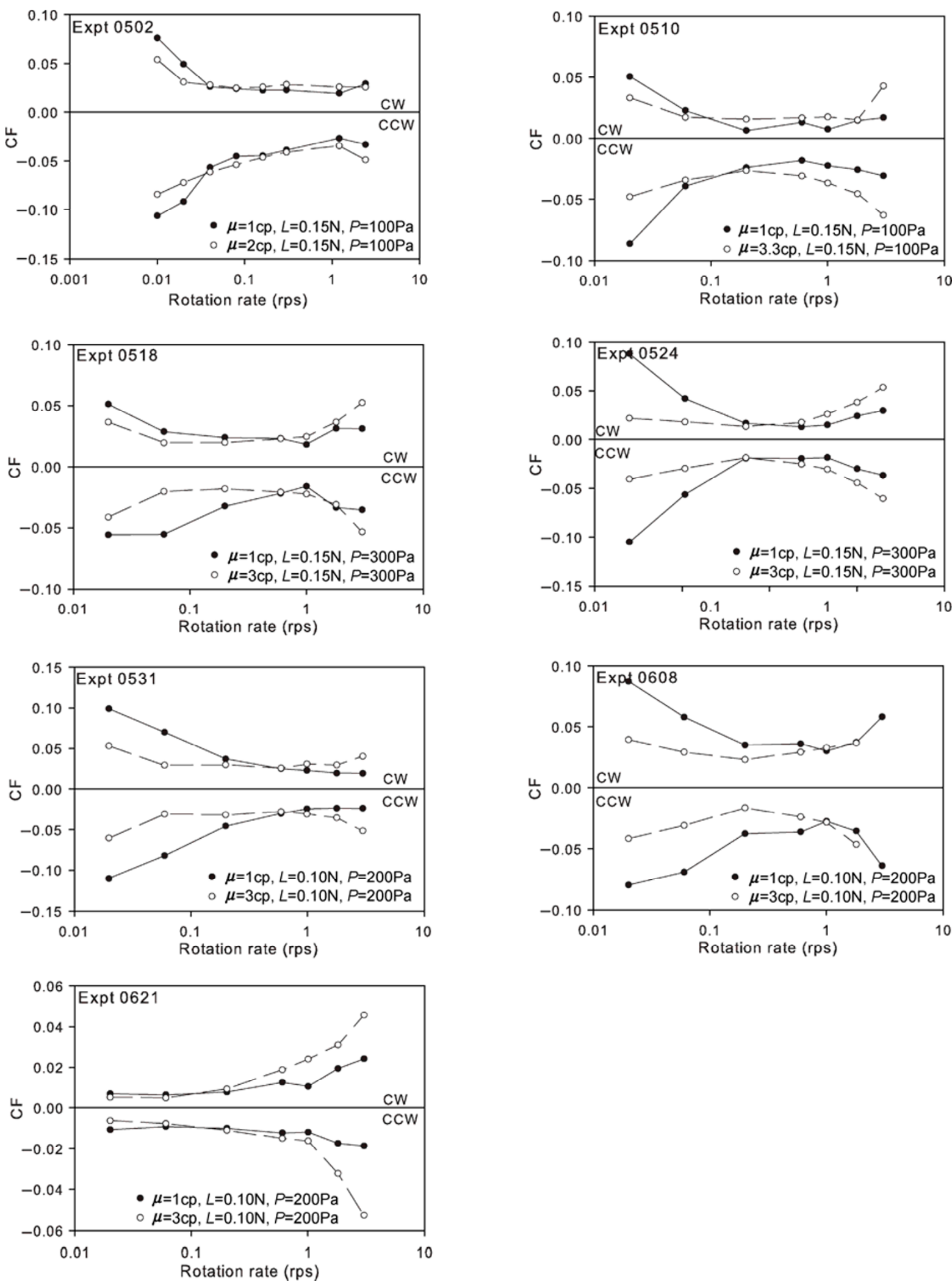

Fig. 4 CF of tissue rotating on glass under loads $(L)$ of 0.10 or $0.15 \mathrm{~N}$ with saline lubricants of viscosity $(\mu)$ of 1,2 , or $3.3 \mathrm{cp}$. Rotation alternated direction as in Fig. 3. 

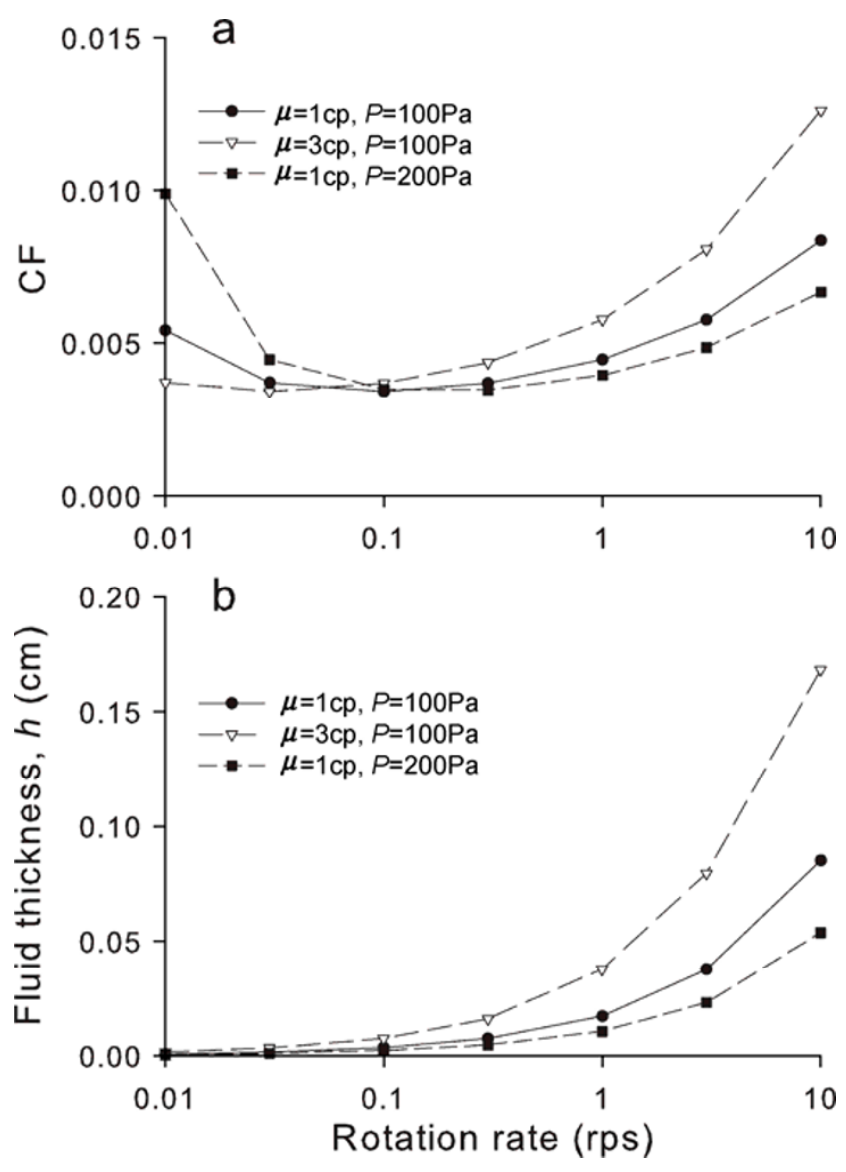

Fig. 5 Simulations of steady state CF (a) and fluid thickness (b) as a function of rotation rate with pressure load $(P)$ of 100 or 200 $\mathrm{Pa}$ and lubricant viscosity $(\mu)$ of 1 or $3 \mathrm{cp}$.

at high speeds without changing CF over the entire mid-range of rotation rates (compare with Fig. 3). Increasing viscosity increases $h$ and reduces $\mathrm{CF}$ at low speeds while increasing $\mathrm{CF}$ at high speeds, reproducing the experimental results in Fig. 3.

\subsection{Decay of frictional transients at the onset of rotation at various rates}

\subsubsection{Experimental observations}

Data from 8 experiments in an earlier study of steady state friction [5] were re-analyzed to determine how the rate at which torque decayed from its peak after the onset of rotation varies with speed of rotation. In a top-fixed apparatus, pressure loads of 100-200 Pa were applied $30 \mathrm{~s}$ before the start of rotation. At rotation speeds below $\sim 0.02$ revolutions per second (rps), torque increased progressively during rotation without reaching a maximum during $30 \mathrm{~s}$ rotation. At rotation rates of $0.05-0.8 \mathrm{rps}$, peak torque was reached soon after the onset of rotation and then decayed to the steady state value, this decay being faster at higher rotation rates (Fig. 6(a)). The normalized rate of transient decay (the characteristic slope of torque decrease with time after peak torque divided by the peak-steady state torque difference) was significantly correlated with rotation rate (Fig. 7(a)).
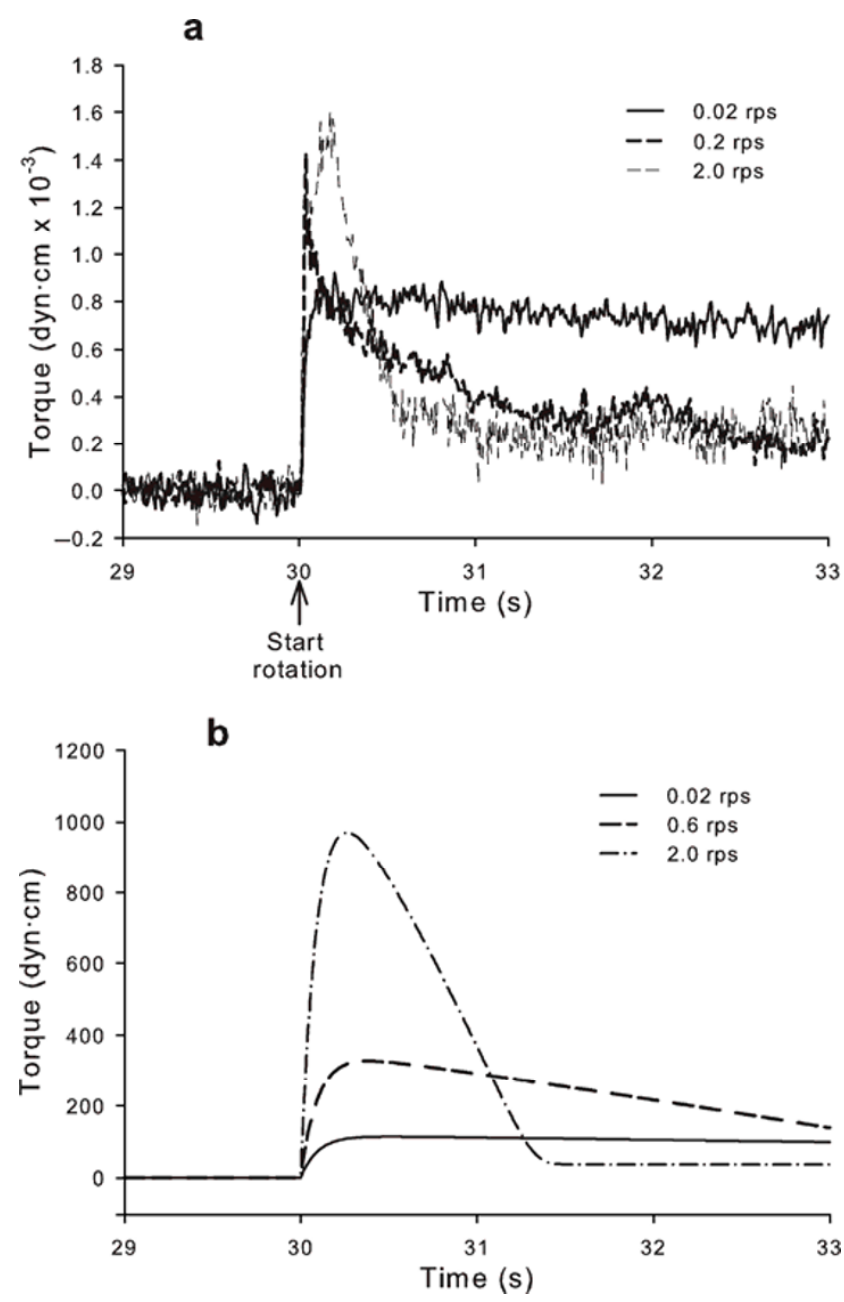

Fig. 6 (a) An example of the torque transients at the onset of rotation at various rates. The peak torque was greater and the decay of the torque transient was quicker at higher rotation rates. (b) Simulated transients at the start of rotation. There is a greater rate-dependence of peak torque in the simulations than in the experimental results. We speculate that deformation of mesothelial tissue by stresses at the onset of rotation reduce the initial torque and redistribute fluid beneath the tissue, whereas peak torques in the simulation are not limited by this mechanism. 


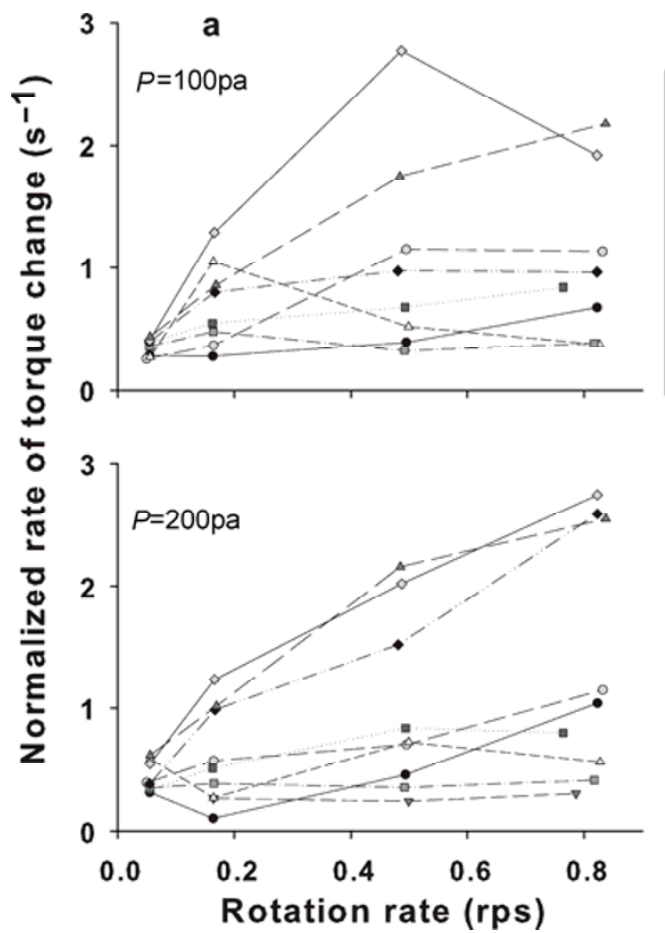

b

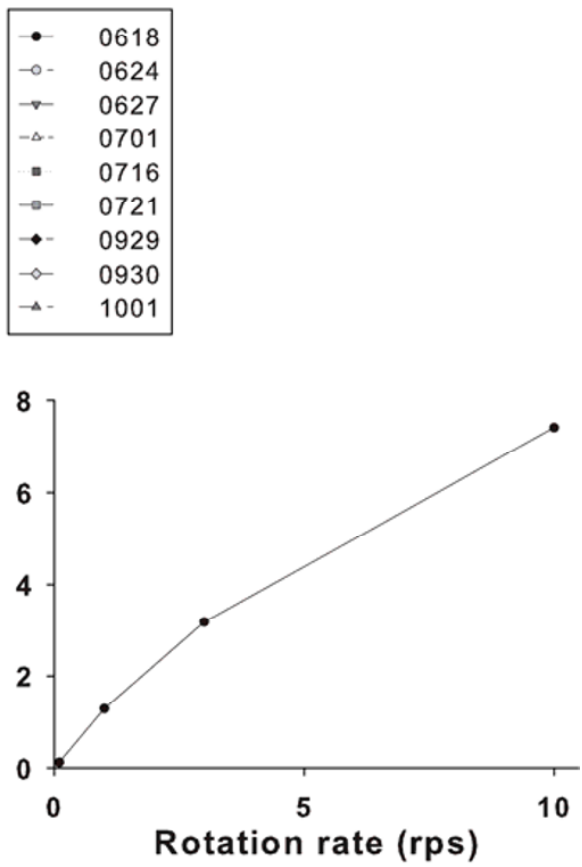

Fig. 7 (a) Characteristic rate of decrease from peak torque normalized by the peak-to-steady state difference in torque at various rotation rates under normal stress of 100 or $200 \mathrm{~Pa}$ in 8 experiments. The normalized rate of torque decrease was significantly related to rotation rate in 5 of 8 experiments and in the group as a whole (ANOVA, $p<0.0001$ ). The rate of decay was also related to the individual tissue preparation $(P<0.0001)$ and pressure $(p=0.0054)$. (b) Simulation: normalized rate of torque decrease after the onset of rotation in simulations at various speeds after a squeeze-out period of $30 \mathrm{~s}$. Normalized decay rate was calculated as the inverse of the initial half-time of the decay. Increasing rotation rate increases the rate of torque decay from the peak to the steady state value.

\subsubsection{Model simulations and mechanism}

The model simulations (Figs. 6(b), 7(b)) started with an initial fluid thickness of $0.1 \mathrm{~mm}$ and a $30 \mathrm{~s}$ period with 100 Pa pressure load to cause squeeze-out before rotation. At the onset of rotation at lower rotation rates, torque increased relatively slowly to a peak and decreased slowly to the steady state value, whereas at higher rates, torque increased and decreased more rapidly. Peak torque and the rate of decay of torque were greater at higher rotation rates because the shear stress and lifting force due to hydrodynamic pumping pressure were greater, and higher rates of fluid thickening increased $h$ and reduced torque more quickly at higher rotation rates. As in the experiments, the onset of rotation at rates below 0.04 rps caused a progressive increase in torque (and $h$ ) without a subsequent torque decrease, because $h$ had not decreased to its steady state value in the $30 \mathrm{~s}$ before rotation began.

\subsection{Transient peaks in friction after different periods of squeeze-out}

\subsubsection{Experimental observations}

In six experiments with the top-fixed apparatus, we measured peak torque at the onset of rotation after periods without rotation ranging from $0.1 \mathrm{~s}$ to $128 \mathrm{~s}$. Rotation rates ranged from 0.002 to 2 rps at pressure loads of 100 or $200 \mathrm{~Pa}$. The peak torque increased with increasing length of the preceding period without rotation in all runs in all experiments.

The data were fit to an equation derived from the rudimentary squeeze-out model described in Section 2.4, together with the additional assumption that the initial peak force is inversely proportional to the thickness of the fluid layer at the onset of rotation. Without rotation, thickness decreases from the steady state thickness at the end of the previous rotation due to squeeze-out. As given by Eq. (8), the height 
of the tissue disk above the bottom plate $(h)$ changes at the rate $\frac{\mathrm{d} h}{\mathrm{~d} t}=\frac{2 h^{3} F_{\mathrm{n}}}{3 \pi \mu R^{4}}$, where $F_{\mathrm{n}}$ is the normal force applied, $R$ is the disk radius, and $\mu$ is fluid viscosity. Integration yields $h(t)=\frac{2 F_{\mathrm{n}}}{3 \pi \mu R^{4} \sqrt{t+t_{\mathrm{i}}}}$ where $t_{\mathrm{i}}$ is the time that would have been required for squeezeout to reduce $h$ from an initial large value to its previous steady state value, which in turn is the initial value during pure squeeze-out. The equation of peak torque has the form,

$$
\text { PeakTorque }=\mathrm{k} \sqrt{t+t_{\mathrm{i}}}
$$

where $\mathrm{k}$ is a constant. Figure 8 shows two representative experimental results.

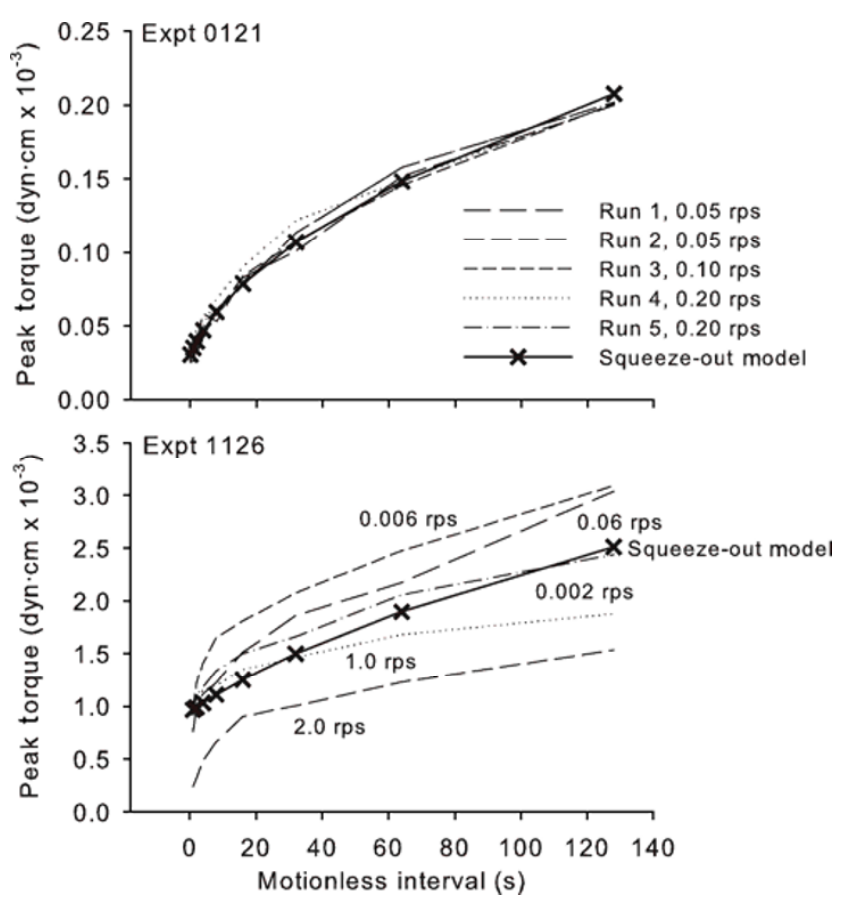

Fig. 8 Peak torque in a top-fixed apparatus at the onset of rotation after various intervals without rotation. In Expt 0121, pressure was $200 \mathrm{~Pa}$ and rotation rates were $0.05,0.1$, and $0.2 \mathrm{rps}$. One squeeze-out model simulation (Eq. (1)) fits all the data relatively well for these 5 runs with a limited range of rotation rate. The constant $t_{\mathrm{i}}$ (see Section 3.3.1) ranged from 2.1 to $3.7 \mathrm{~s}$ in theses experiments. In Expt 1126, pressure was $100 \mathrm{~Pa}$, and there was a wider range of rotation rates. Although Eq. (1) continued to fit individual curves relatively well, no single simulation could fit all curves. The large difference in the toque magnitudes between the two experiments is typical of previous results [5], and we speculate that it arises from differences in surface topography and mechanical properties of individual tissue preparations.

\subsubsection{Model simulations and mechanism}

The simulation results were similar to the experimental data. Figure 9 shows the peak torque and thickness of the liquid layer at the end of intervals without motion. The basic squeeze-out model (Eq. (1)) fits data at individual speeds well.

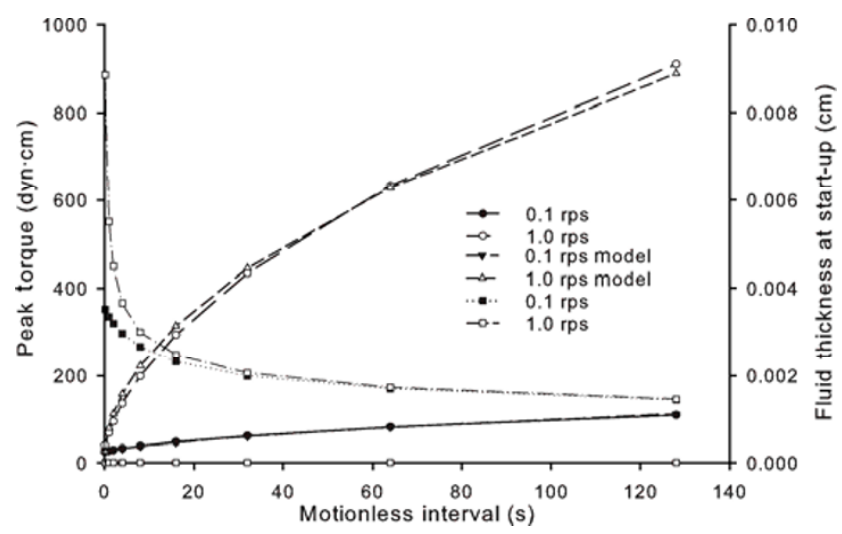

Fig. 9 Simulated peak torques following intervals without rotation of various durations (left axis) and $h$ values at the end of the intervals (right axis) with rotation rates of 0.1 or $1 \mathrm{rps}$ with a pressure load of $100 \mathrm{~Pa}$. The progressive increase in torque with increasing time without motion can be attributed to the progressive reduction in $h$ due to squeeze-out. Note that the initial difference in $h$ at the two rotation rates almost disappears by $128 \mathrm{~s}$, and the curves rapidly converge, consistent with the $h^{3}$ dependence of the squeeze-out rate (Eq. (9)).

\subsection{Sinusoidal rotation}

\subsubsection{Experimental observations}

Sinusoidal rotation was applied in 9 experiments with the top-fixed apparatus at frequencies from 0.005 to 1.25 , with peak rotation rates from 0.006 to $1.25 \mathrm{rps}$ and pressures of 100 or $200 \mathrm{~Pa}$. Figure 10 shows examples of the force-displacement and force-velocity plots at high and low frequencies in one experiment. At higher frequencies, torque vs. displacement plots were rounded (Fig. 10(a)) and force vs. velocity plots were nearly linear (Fig. 10(b)), indicating that torque varied with velocity. By contrast, at low frequencies, force displacement plots were relatively rectangular (Fig. 10(c)), indicating that torque was relatively constant with speed, changing sign with direction of rotation (Fig. 10(d)). 

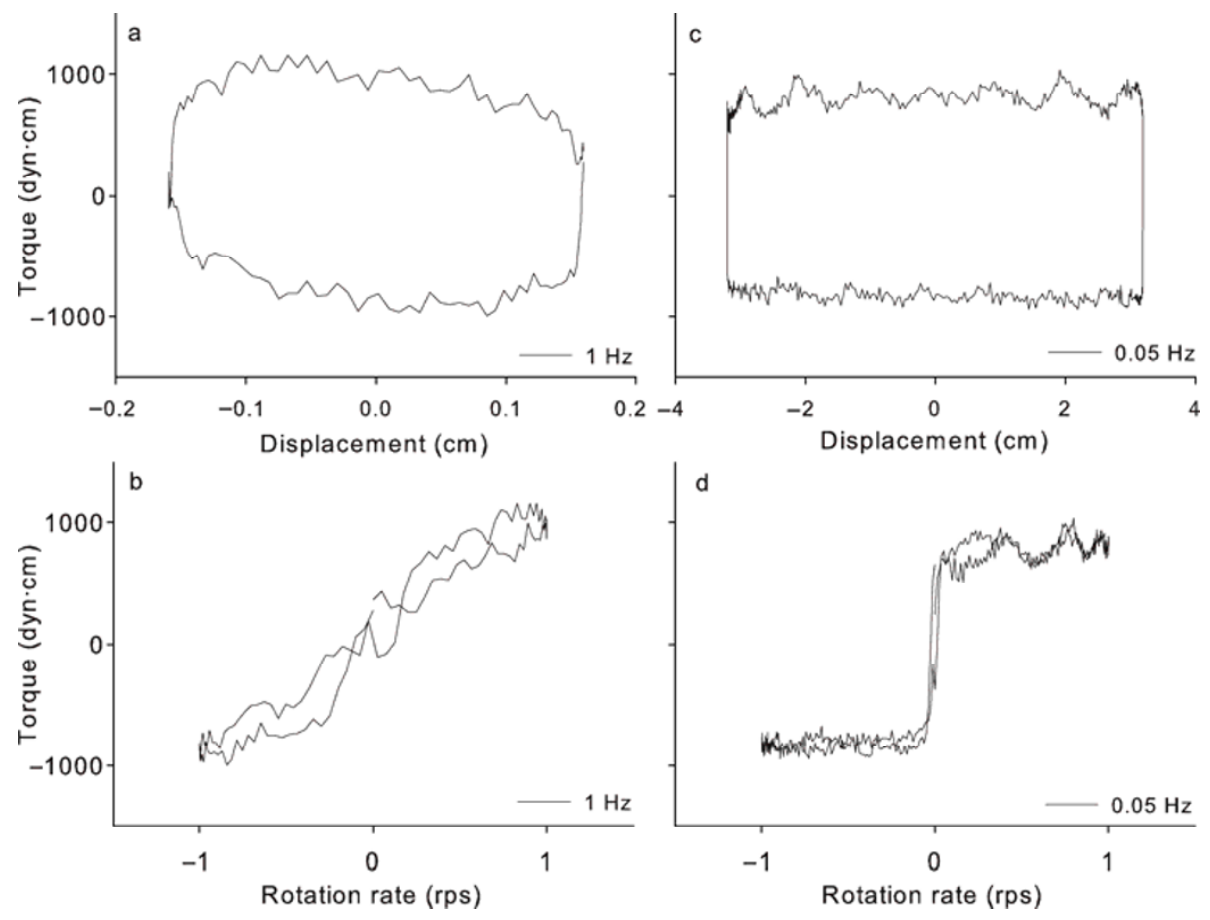

Fig. 10 Torque during sinusoidal oscillation at $1 \mathrm{~Hz}(\mathrm{a}, \mathrm{b})$ and $0.05 \mathrm{~Hz}$ (c, d), with $1 \mathrm{rps}$ peak velocity and a pressure load of $100 \mathrm{~Pa}$. Torque was nearly in phase with velocity at the higher frequency and nearly invariant with velocity at the lower frequency. These results were typical.

\subsubsection{Model simulations and mechanism}

Model simulations showed similar variations in the shapes of torque-displacement and force-velocity characteristics with frequency. At high frequencies and velocities, torque is nearly proportional to velocity (Figs. 11(a) and 11(b)). This can be attributed to the fact that $h$ is relatively constant because the short period of the oscillatory cycle does not permit substantial squeeze-out (Fig. 11(c)).

At low frequencies, (Figs. 11(d) and (e)), torque was nearly independent of velocity and the forcedisplacement characteristic was more rectangular. At high velocities and low frequencies, the relatively constant torque can be attributed to relatively large variation in both $h$ and $h_{\min }$ due to the dynamic equilibrium between hydrodynamic pumping and squeeze-out that stabilizes shear stress as velocity changes (Figs. 11(c) and (f)). The asymmetry of the torque-displacement curves at high frequency and the cusps on torque-displacement and torque-velocity curves at low frequency (Figs. 11(a), (d), and (e)) are caused by the transiently low values of $h$ following squeeze-out during the preceding low velocity
(Figs. 11(c) and (f)). These asymmetries were sometimes observed in the experimental data (note the similar asymmetry in Fig. 10(a)).

\section{Discussion}

Here, we have interpreted steady state and transient behavior of tissues sliding in saline with a model that synthesizes physical principles and phenomena of elastohydrodynamic lubrication. This analysis extends earlier theoretical work suggesting a hydrodynamic mechanism that could explain a relatively constant CF with decreasing velocity [12] to transient and nonsteady state sliding. Although elastohydrodynamic mechanisms are able to explain many experimental phenomena, their importance in mesothelial lubrication in vivo is debated.

Conventionally, a transient peak in force at the onset of sliding has been interpreted as implying that the surfaces initially in contact exhibit static friction [13]. Force increases with increasing elastic deformation until the surfaces break free and slide at a lower frictional force in the steady state; if contact returns 

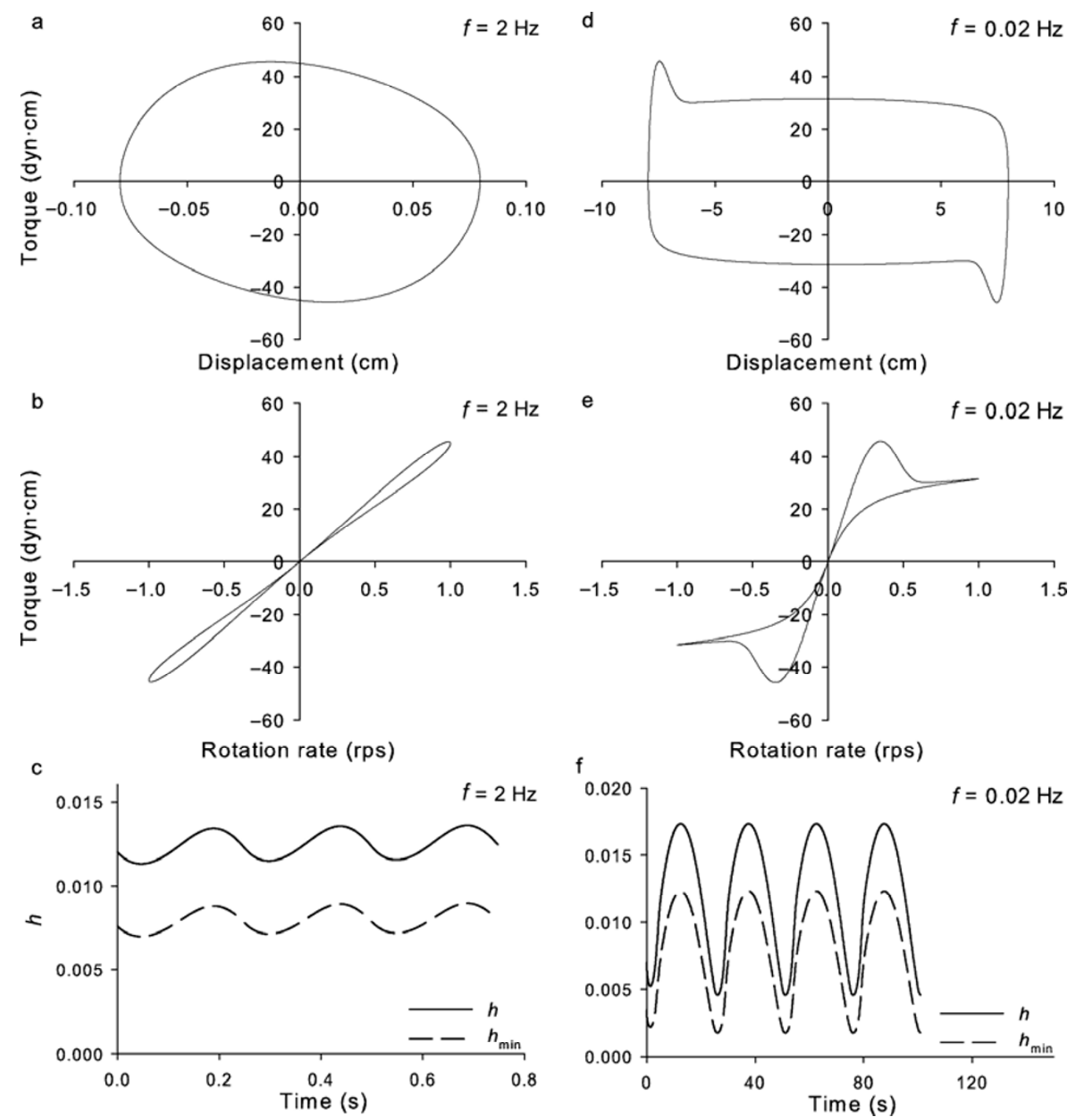

Fig. 11 Simulations of oscillatory rotation at 2 or $0.02 \mathrm{~Hz}$ with a load of $100 \mathrm{~Pa}$ and velocity amplitude of $1 \mathrm{rps}$ (See Section 3.4.2).

and this cycle repeats, it constitutes the classical "stick-slip" behavior. By contrast, in our simulations surfaces are never in contact, and there is no elastic deformation caused by shear stress of tissues before sliding begins. Instead, the transient variations in torque are due to history-dependent variations in the thickness of the lubricating fluid layer, which is decreased by squeeze-out, increased by hydrodynamic pumping, and influenced by elastic smoothing of the surface roughness. Without motion, the thickness of the liquid layer decreases at a rate inversely proportional to the square root of time.

The coefficients of friction observed in the experiments described are variable, and, in general, much greater than those in the model simulation. In a previous study [5], we noted a remarkable variability among tissue specimens in the steady state torque and patterns of torque variation with velocity, and we speculated that such variability is due to topographical features peculiar to individual tissue specimens that augment or diminish the load bearing effects of hydrodynamic lubrication. We infer that the model's regular and homogeneous surface unevenness results in lower shear stress with relatively homogeneous fluid thickness. Alternatively, the higher torques in experimental preparations may reflect regions of contact between surfaces. This is the view held by Agostoni, D'Angelo and others [3,4] who have 
interpreted the velocity-invariant friction observed with mesothelial tissues during rectilinear sliding experiments as evidence for boundary lubrication. However, the velocity-dependent behavior we observed during rotational tribometry [5] was reproduced in experiments using rectilinear tribometry of tissue on glass (unpublished), suggesting that the difference in speed-dependence of CF is not due to rectilinear versus rotational tribometry, but rather to differences in the normal loads and sliding velocities employed.

The three elements of the model are based to varying degrees on theory and experimental observation. Squeeze-out is an obvious phenomenon when lubricated surfaces are pressed together without sliding motion, as described by the physics of a piston sinking under a load (see Section 2.4). Hydrodynamic pumping is based on the two-dimensional lubrication theory [11], whereby fluid thickness is increased between sliding surfaces that are nearly parallel. In three dimensions, hydrodynamic pumping is a general phenomenon exhibited by soft shapes sliding in lubricant [9], and has been demonstrated during rotational sliding in biomechanical experiments [6] and in finite element models [8]. Elastohydrodynamic pumping lacks a rigorous general mathematical description, as does hydrodynamic smoothing. The biological relevance of elastic smoothing of mesothelial tissue surfaces has been recently explored through mechanical surface characterization [14].

In conclusion, the friction of mesothelial tissues during steady state and time-varying sliding can be largely reproduced by a synthesis of three phenomena characteristic of elastohydrodynamic lubrication. These phenomena are thinning of the lubricating fluid layer by squeeze-out under a normal load, thickening of the fluid layer through hydrodynamic pumping, and elastic smoothing of surface unevenness by local hydrodynamic stresses. These phenomena provide a plausible mechanism to explain tribological experiments in sliding mesothelial tissues, but they do not preclude the possibility of other mechanisms, including tissue-tissue contact.

\section{Conflict of interest statement}

The authors have no financial or personal relationship with other people or organizations that could inappropriately influence this investigation.

\section{Acknowledgements}

The authors are indebted to Richard E. Brown for physiological insights and experimental data presented here, and we thank Jae Hun Kim for help in revision. The work is supported by grant HL-63737 from the National Institutes of Health.

Open Access: This article is distributed under the terms of the Creative Commons Attribution Noncommercial License which permits any noncommercial use, distribution, and reproduction in any medium, provided the original author(s) and source are credited.

\section{References}

[1] Brandi G. Determinazione del coefficiente di attrito statico tra le due pleure. Boll Soc Ital Biol Sper 46(8): 427-429 (1970)

[2] Brandi G. Frictional forces at the surface of the lung. Bull Physiopathol Respir (Nancy) 8(2): 323-336 (1972)

[3] D'Angelo E. Stress-strain relationships during uniform and non uniform expansion of isolated lungs. Respir Physiol 23(1): 87-107 (1975)

[4] D'Angelo E, Loring S H, Gioia M E, Pecchiari M, Moscheni C. Friction and lubrication of pleural tissues. Respir Physiol Neurobiol 142(1): 55-68 (2004)

[5] Loring S H, Brown R E, Gouldstone A, Butler J P. Lubrication regimes in mesothelial sliding. J Biomech 38(12): 2390-2396 (2005)

[6] Lin J L, Moghani T, Fabry B, Butler J P, Loring S H. Hydrodynamic thickening of lubricating fluid layer beneath sliding mesothelial tissues. J Biomech 41(6): 1197-1205 (2008)

[7] Gouldstone A, Brown R E, Butler J P, Loring S H. Elastohydrodynamic separation of pleural surfaces during breathing. Respir Physiol Neurobiol 137(1): 97-106 (2003)

[8] Moghani T, Butler J P, Lin J L, Loring S H. Finite element simulation of elastohydrodynamic lubrication of soft biological tissues. Comput Struct 85(11-14): 1114-1120 (2007)

[9] Skotheim J M, Mahadevan L. Soft lubrication: The elastohydrodynamics of nonconforming and conforming contacts. Physics of Fluids 17(9): 092101-092123 (2005)

[10] Butler J P, Huang J, Loring S H, Lai-Fook S J, Wang P M, Wilson T A. Model for a pump that drives circulation of pleural fluid. J Appl Physiol 78(1): 23-29 (1995) 
[11] Batchelor G K. An Introduction to Fluid Dynamics. Cambridge, UK: Cambridge University Press, 1967.

[12] Butler J P, Loring S H. A potential elastohydrodynamic origin of load-support and coulomb-like friction in lung/chest wall lubrication. J Tribol 130(4): 041201 (2008)

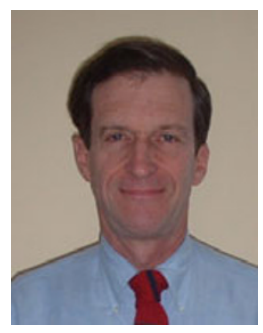

Stephen H. LORING received his M.D. from Harvard Medical School in 1973 and joined the Physiology Department at Harvard School of Public in 1977. In 1991 he joined the Department of Anesthesia and

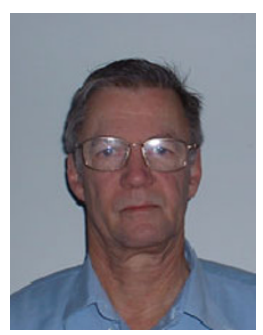

James P. BUTLER received his Ph.D. in physics from Harvard Univ. in 1974, and for the past 4 decades has worked in a wide variety of applications of physics to respiratory physiology. At the whole organ
[13] Bowden F P, Talbor D. The Friction and Lubrication of Solids. Oxford, UK: Oxford University Press, 2001.

[14] Kim J H, Butler J P, Loring S H. Influence of the softness of the parietal pleura on respiratory sliding mechanisms. Respir Physiol Neurobiol 177(2): 114-119 (2011)

Critical Care at Beth Israel Deaconess Medical Center and Harvard Medical School, where his current position is Scientific Director of Respiratory Medicine. His research has centered on respiratory physiology and medicine and the biomechanics and physiology of the pleural space.

and integrated level, his particular interests include lung mechanics, gas exchange, aerosol transport, and sleep disordered breathing; at the cellular and tissue level he works in the rheological properties of single cells, migrating monolayers, and elastohydrodynamic fluid/tissue interactions. 